\title{
Publisher Correction: An electrochemical thermal transistor
}

\author{
Aditya Sood (1) 1,2,13, Feng Xiong (1) 1,3,9,13, Shunda Chen ${ }^{4}$, Haotian Wang (1) 1,10, Daniele Selli ${ }^{5,11}$, Jinsong Zhang ${ }^{1}$, \\ Connor J. McClellan ${ }^{3}$, Jie Sun ${ }^{1,12}$, Davide Donadio ${ }^{4,6}$, Yi Cui $^{1,7 \star}$, Eric Pop (D) ${ }^{1,3,8 \star}$ \& Kenneth E. Goodson ${ }^{1,2 \star}$
}

Correction to: Nature Communications https://doi.org/10.1038/s41467-018-06760-7, published online 30 October 2018.

The original version of this Article contained an error in Fig. 2a in which the label on the $y$-axis incorrectly read ' $\mathrm{Al} / \mathrm{MoS}_{2} \mathrm{OiS} / 2$ ' rather than the correct ' $\mathrm{Al} / \mathrm{MoS}_{2} / \mathrm{SiO}_{2}$ '.

Further, the original version contained an error in the last sentence of the seventh paragraph of the Discussion which incorrectly read 'Reducing the temperature swing can have a significant effect on device reliability as the relationship between $\Delta T$ and the number of cycles to failure $N_{\mathrm{f}}$ is strongly non-linear ${ }^{38}, N_{\mathrm{f}} \sim \Delta T^{-3,5}$ '. The correct version states ' $\Delta T^{-3.5}$ ' in place of ' $\Delta T^{-3,5}$ '.

This has been corrected in both the PDF and HTML versions of the Article.

Published online: 27 September 2019

\begin{abstract}
(c) (i) Open Access This article is licensed under a Creative Commons Attribution 4.0 International License, which permits use, sharing, adaptation, distribution and reproduction in any medium or format, as long as you give appropriate credit to the original author(s) and the source, provide a link to the Creative Commons license, and indicate if changes were made. The images or other third party material in this article are included in the article's Creative Commons license, unless indicated otherwise in a credit line to the material. If material is not included in the article's Creative Commons license and your intended use is not permitted by statutory regulation or exceeds the permitted use, you will need to obtain permission directly from the copyright holder. To view a copy of this license, visit http://creativecommons.org/licenses/by/4.0/.
\end{abstract}

(C) The Author(s) 2019

\footnotetext{
${ }^{1}$ Department of Materials Science and Engineering, Stanford University, Stanford, CA 94305, USA. ${ }^{2}$ Department of Mechanical Engineering, Stanford University, Stanford, CA 94305, USA. ${ }^{3}$ Department of Electrical Engineering, Stanford University, Stanford, CA 94305, USA. ${ }^{4}$ Department of Chemistry, University of California, Davis, CA 95616, USA. ${ }^{5}$ Max Planck Institute for Polymer Research, Ackermannweg 10, D-55128 Mainz, Germany. ${ }^{6}$ Ikerbasque, Basque Foundation for Science, E-48011 Bilbao, Spain. ${ }^{7}$ Stanford Institute for Materials and Energy Science, SLAC National Accelerator Laboratory, Menlo Park, CA 94025, USA. ${ }^{8}$ Precourt Institute for Energy, Stanford University, Stanford, CA 94305, USA. ${ }^{9}$ Present address: Department of Electrical and Computer Engineering, University of Pittsburgh, Pittsburgh, PA 15261, USA. ${ }^{10}$ Present address: Department of Chemical and Biomolecular Engineering, Rice University, Houston, TX 77005, USA. ${ }^{11}$ Present address: Dipartimento di Scienza dei Materiali, Universita di Milano-Bicocca, 20125 Milano, Italy. ${ }^{12}$ Present address: School of Chemical Engineering and Technology, Tianjin University, 300350 Tianjin, China. ${ }^{13}$ These authors contributed equally: Aditya Sood, Feng Xiong. *email: yicui@stanford.edu; epop@stanford.edu; goodson@stanford.edu
} 